\title{
Postoperative Nausea \& Vomiting in Malawi
}

\author{
Samson Mndolo, Kai Jung, Gregor Pollach* \\ Department of Anaesthesia and Intensive Care, College of Medicine, University of Malawi, Blantyre, Malawi \\ Email: "gipi.bc62@yahoo.de
}

Received 5 February 2014; revised 4 March 2014; accepted 3 April 2014

Copyright (C) 2014 by authors and Scientific Research Publishing Inc.

This work is licensed under the Creative Commons Attribution International License (CC BY). http://creativecommons.org/licenses/by/4.0/

(c) (i) Open Access

\section{Abstract}

Background: Postoperative nausea and vomiting are common complications of anaesthesia and surgery. Known risk factors include motion sickness, migraine, gender and types of surgery. Other possible risk factors for postoperative nausea and vomiting are ethnicity and genetics surgery. Objective: The main objective of the study was to describe factors associated with postoperative nausea and vomiting among adult Malawians. Methods: This was a prospective observational study. 138 adult patients were recruited into the study. Data were collected using a predesigned questionnaire. Patients were followed up to 18 hours postoperatively and any episodes of postoperative nausea and vomiting were noted. Results: A total of 138 patients were enrolled in the study. 78 were female $(56.5 \%)$ and 60 were males $(43.5 \%)$. The ages ranged from 18 to 87 years. The mean age was 36.9 years. The overall incidence of postoperative nausea and vomiting was 29.6\%. It was higher among women than men. Patients with motion sickness had the highest incidence of postoperative nausea and vomiting (78.6\%) followed by those with migraine $(73.3 \%)$. Patients whose intraoperative systolic blood pressure fell $<80 \mathrm{mmHg}$ had an incidence of $71.4 \%$ and those who received postoperative opioids had an incidence of $37.7 \%$. Conclusions: Patients with a history of migraine, motion sickness, whose intraoperative blood pressures fall below a systolic of $80 \mathrm{mmHg}$ and who receive postoperative opioids are at an increased risk for postoperative nausea and vomiting.

\section{Keywords}

PONV, Postoperative Nausea and Vomiting, Malawi, Africa, Ketamin, Pethidine

\section{Introduction}

Postoperative nausea and vomiting are common postoperative complications of anaesthesia and surgery [1]. It is considered as a common cause of morbidity following anaesthesia and reduces patients' satisfaction [2]. It is generally known to affect patients undergoing all types of anaesthesia [3].

\footnotetext{
*Corresponding author.
} 
Although postoperative nausea and vomiting have been extensively studied, not much has been done in sub Saharan Africa. The studies which have been done have yielded mixed results. In some studies, the overall incidence reported has been much lower than that reported in Caucasians while others have reported similar results.

The only studies to our knowledge were conducted in Ghana, Nigeria, the Republic of South Africa and Uganda [4]-[7].

So far no study has been conducted in Malawi on this subject. Could Malawians have similar incidences as other parts of Africa? Africa is a multi-ethnic continent and it could not be surprising to find totally different results in areas sharing political boundaries.

Like many African countries with limited health budgets, Malawi cannot afford universal prophylactic antiemetic medication or treatment. This is why it is necessary to have data to appreciate whether postoperative nausea and vomiting are problems worthy of the attention of policy makers.

\section{Methods}

This was a prospective observational study. The study was conducted at Queen Elizabeth Central Hospital in Blantyre, Malawi. Queen Elizabeth Central is the main teaching and referral hospital in Malawi. Patients were followed from the wards then theatre, recovery room and back to the wards.

The study was approved by the college of medicine research and ethics committee (COMREC) on $20^{\text {th }}$ June 2013. To maintain confidentiality no names were recorded. The study recruited patients aged 18 years and above presenting for elective surgery who met the inclusion criteria. All patients were indigenous Malawians.

A sample size was calculated using a marginal of error of 5\%, CI 95\% an estimated population incidence of postoperative nausea and vomiting of $10 \%$.

$$
S S=\frac{Z^{2} \times(p) \times(1-p)}{C_{2}}
$$

SS = Sample Size;

$Z=Z$-value (i.e. 1.96 for a 95 percent confidence level);

$P=$ estimated population incidence of PONV, expressed as decimal, 0.1 in this case;

$C=$ Confidence interval, expressed as decimal, 0.05 in this case. Therefore the calculated sample size was 138 .

Consecutive sampling was used i.e. participants were recruited according to the order in which they appeared on the theatre list.

The first one was approached first, and then so on so forth until the required sample size of 138 was achieved.

Patients were first seen on the ward 12 to 18 hours preoperatively. The purpose of the study was explained and written consent was obtained. They were then interviewed on the ward according to a predesigned data sheet. Each interview took 5 to 7 minutes. Data obtained included biographic data, and presence or absence of certain preoperative risk factors for postoperative nausea and vomiting.

Patient received either a general or regional anaesthetic. In theatre routine monitoring was applied and baseline vital signs were recorded. Intravenous cannulas were checked for patency and reinserted where necessary. The start of anaesthesia was defined as the time at which all routine anaesthetic monitors were applied. No patient received any premedication preoperatively. All patients who had general anaesthesia were preoxygenated for three minutes prior to induction. Induction drugs were chosen according to the preference of anaesthetist or anaesthetic clinical officer responsible for the case.

Each patient had an intraoperative record completed. Data like fluids given; drugs given; intraoperative blood pressures were all noted on this record.

Data were also extracted from patients' medication charts which showed medications given postoperatively. Postoperatively patients were visited and any episodes of nausea, vomiting or both were recorded. Patients were discharged from follow up 18 hours postoperatively.

All data sheets were checked for completeness before patients were discharged from follow up. All patients with either nausea or vomiting were referred to the clinicians responsible for the particular wards for treatment.

Data were entered into a computer using STATA version 12. Pearson chi square test was used to test association. Logistic regression was used to determine odds ratios and prognostic factors of postoperative nausea and vomiting. 


\section{Results}

Data were collection from the $21^{\text {st }}$ of June, 2013 to the $25^{\text {th }}$ of July 2013. 146 consecutive patients met the inclusion criteria, gave consent and were enrolled in the study. 8 of these had incomplete data and were therefore discarded. 138 patients were included in analysis. Of these, 78 were female (56.5\%) and 60 were males (43.5\%) (Table 1). The ages ranged from 18 to 87 years. The mean age was 36.9 years with a standard deviation of 14.21 (Table 2).

Description of the population.

86 of 138 patients had general anaesthesia and the remainder had regional anesthesia. Of the patients who received general anaesthesia, 71 (82.5\%) of them were induced with thiopentone. Most patients who had general anaesthesia, 82 of 86 (95.3\%) received pethidine as a form of intraoperative analgesia. Most patients, 121 (87.7\%) of 138 had an introperative systolic blood pressures of above $90 \mathrm{mmHg} 87$ (63\%) of patients received between $1000 \mathrm{ml}$ and $2000 \mathrm{ml}$ of fluids intraoperatively, 23 (16.7\%) had less than $1000 \mathrm{ml}$ and 28 (20.2\%) had over $2000 \mathrm{ml}$ of intravenous fluids. 90 (65.2\%) of the patients received opioids postoperatively; the rest had other forms of analgesia.

Most procedures lasted more than one hour, 87 (63\%) of the 138. Gynaecological surgery was the commonest procedure performed in the study population (21\%) and Ear, Nose and Throat surgery was the least, 12 (8.7\%).

The overall incidence of postoperative nausea and vomiting was $29.7 \%$ (41 cases out of 138). The youngest age group had the highest incidence while the oldest had the lowest. Patients with a history of motion sickness had the highest incidence 78.6\%, $(p<0.0001)$ followed by those with a history of migraine; $73.3 \%(p<0.0001)$ (Table 3).

Patients who had a systolic blood pressure of less than $80 \mathrm{mmHg}$ intraoperatively had a higher incidence of postoperative nausea and vomiting as opposed to those whose systolic blood pressures were $80-90 \mathrm{mmHg}$ or higher; $71.4 \%$, 30\% and $27.2 \%$ respectively ( $<<0.035)$.

Postoperative use of opioids also increased the incidence of postoperative nausea and vomiting; $37.8 \%$ in patients who had opioids and $14.6 \%$ in those who did not $(\mathrm{p}<0.015)$.

Table 1. Gender distribution.

\begin{tabular}{ccc}
\hline Gender & Frequency & Percent \\
\hline Male & 60 & 43.5 \\
Female & 78 & 56.5 \\
\hline
\end{tabular}

Table 2. Age distribution.

\begin{tabular}{ccc}
\hline Age group in years & Frequency & Percent \\
\hline 20 & 9 & 6.5 \\
$20-30$ & 45 & 32.6 \\
$31-40$ & 40 & 29 \\
$41-50$ & 28 & 11.6 \\
$>50$ & 16 & 100 \\
\hline
\end{tabular}

Table 3. Distribution of preoperative risk factors.

\begin{tabular}{ccc}
\hline Risk factor & Yes & No \\
Migraine & $15(10.9 \%)$ & $123(89.1 \%)$ \\
Motion sickness & $14(10.1 \%)$ & $124(89.9 \%)$ \\
Smoking & $13(9.4 \%)$ & $125(90.6 \%)$ \\
\hline
\end{tabular}




\section{Discussion}

This study has shown that the incidence of postoperative nausea and vomiting at Queen Elizabeth Central Hospital in Blantyre, Malawi is $29 \%$. This is not very different from what was found among black South Africans. Rodseth et al. found an incidence of postoperative nausea and vomiting of $27 \%$ among black South African [6]. However a study by Ssebuufu in Uganda found a $40 \%$ incidence of postoperative nausea and vomiting which is higher than our findings [7]. The incidence in this study is lower than in the Ugandan study possibly because in this study follow up of patients stopped at 18 hours as opposed to the Ugandan study in which patients were followed up to 24 hours postoperatively.

Other authors have suggested an ethnic predisposition to postoperative nausea and vomiting [6] [8]. One would argue that Malawians are ethnically more similar to black South Africans than Ugandans as evidenced by the presence of ethnic groups in Malawi that emigrated from the Republic of South Africa in the $17^{\text {th }}$ century [9].

Females had a higher incidence of postoperative nausea and vomiting compared to men. This is similar to what both Koivuranta et al. and Gan et al. demonstrated that female gender increases the risk for postoperative nausea and vomiting [10] [11].

Patients with motion sickness and migraine reported highest incidences of postoperative nausea and vomiting. This is also consistent to findings Koivuranta who demonstrated that migraine and motion sickness were found to be significant [10] preoperative factors for postoperative nausea and vomiting.

Incidence was higher among non smokers than smokers. This is consistent with what others have found that non-smoking status is a risk factor for post operative nausea and vomiting. In a study by Chimbira it was noted that those who did not smoke were more likely to develop postoperative nausea and vomiting [12].

The incidence of postoperative nausea and vomiting was highest in the gynaecological population. This was much higher [5] than what was found in Nigeria. Ogochukwa et al. found a 4\% incidence of postoperative nausea and vomiting among gynaecological and obstetric patients. However it is similar to what other studies have demonstrated [13] elsewhere. Despite being described as being particularly emetogenic, abdominal surgery had one of the lowest incidences in this study.

The lowest incidence of postoperative nausea and vomiting was among the obstetric population. This could be explained by the fact that almost all cases were done under regional anaesthesia. It has been demonstrated that regional anesthesia reduces the incidence of postoperative nausea and vomiting [1] [10].

The incidence of postoperative nausea and vomiting was higher in patients who had received intraoperative opioids than those who had ketamine as an analgesic. This is consistent with what was shown in other studies that intraoperative use of opioids increases the incidence [10] of postoperative nausea and vomiting. However this study did not show any significant relationship between intraoperative opioid use and postoperative nausea and vomiting. Many studies have demonstrated a significant relationship between intraoperative opioid [10] [14] use and postoperative and vomiting. The only available opioid during this period was pethidine and that was given. It has been shown than the more potent opioids are more emetogenic one would argue that possibly there could be a higher incidence [15] if more potent had been used.

Patients who had lower intraoperative blood pressure had higher incidence than their counterparts who maintained systolic above $90 \mathrm{mmHg}$. The effects of systolic blood pressure on the incidence of postoperative nausea and vomiting has been investigated [16] before. A study by Pusch et al. showed similar results. In their study they followed 300 women undergoing elective surgery under general anaestheisia. They wanted to find out the effect of dropping systolic pressures $>35 \%$ from baseline on the incidence of postoperative nausea and vomiting. Patients whose blood pressure dropped $>35 \%$ had a higher incidence of postoperative nausea and vomiting than those whose systolic pressures were maintained above that [16].

Patients who received opioids had a higher incidence of postoperative nausea and vomiting than those who did no. This finding is similar to what other authors have [10] demonstrated.

\section{Conclusion and Recommendation}

The study concludes that the postoperative nausea and vomiting are significant problems among Malawians worthy of the attention of anaesthetists and all clinicians involved in the care of surgical patients.

\section{Acknowledgements}

We would like to thank all clinical officers in the department of anaesthesia at Queen Elizabeth Central Hospital 
for making sure that all anaesthetic records were completed.

Special thanks to Fanny Tewesa secretary in the Departments of Anaesthesia for typing and printing the manuscript.

Special thanks to Mr. Andrew Kumitawa for his help with statistical analysis.

\section{References}

[1] Gan, T.J., Meyer, T., Apfel, C.C., Chung, F., Davis, P.J., Eubanks, S. and Watcha, M. (2003) Consensus Guidelines for Managing Postoperative Nausea and Vomiting. Anesthesia \& Analgesia, 97, 62-71. http://dx.doi.org/10.1213/01.ANE.0000068580.00245.95

[2] Kovac, A.L. (2000) Prevention and Treatment of Postoperative Nausea and Vomiting. Drugs, 59, $213-243$. http://dx.doi.org/10.2165/00003495-200059020-00005

[3] Van Den Bosch, J.E., Kalkman, C.J., Vergouwe, Y., Van Klei, W.A., Bonsel, G.J., Grobbee, D.E., et al. (2005) Assessing the Applicability of Scoring Systems for Predicting Postoperative Nausea and Vomiting. Anaesthesia, 60, 323-331. http://dx.doi.org/10.1111/j.1365-2044.2005.04121.x

[4] Amponsah, G. (2007) Postoperative Nausea and Vomiting in Korlebu Teaching Hospital. Ghana Medical Journal, 41, 181-185.

[5] Ugochukwu, O., Adaobi, A., Ewah, R. and Obioma, O. (2010) Postoperative Nausea and Vomiting in a Gynecological and Obstetrical Population in South Eastern Nigeria. Pan African Medical Journal, 7, 6.

[6] Rodseth, R.N., Gopalan, P.D., Cassimjee, H.M. and Goga, S. (2010) Reduced Incidence of Postoperative Nausea and Vomiting in Black South Africans and Its Utility for a Modified Risk Scoring System. Anesthesia \& Analgesia, 110, 1591-1594. http://dx.doi.org/10.1213/ANE.0b013e3181da9005

[7] Ssebuufu, R., Kakande, I. and Okello, M. (2009) Post-Operative Nausea and Vomiting at Mulago Hospital. East and Central African Journal of Surgery, 14, 50-57.

[8] Candiotti, K.A., Birnbach, D.J., Lubarsky, D.A., Nhuch, F., Kamat, A., Koch, W.H. and Andrews, D. (2005) The Impact of Pharmacogenomics on Postoperative Nausea and Vomiting: Do CYP2D6 Allele Copy Number and Polymorphisms Affect the Success. Anesthesiology, 102, 543-549. http://dx.doi.org/10.1097/00000542-200503000-00011

[9] Carruthers, J. (2010) The Changing Shape and Scope of Southern African Historical Studies. South African Historical Journal, 62, 384-393. http://dx.doi.org/10.1080/02582473.2010.493009

[10] Koivuranta, M., Läärä, E., Snåre, L. and Alahuhta, S. (1997) A Survey of Postoperative Nausea and Vomiting. Anaesthesia, 52, 443-449. http://dx.doi.org/10.1111/j.1365-2044.1997.117-az0113.x

[11] Gan, T.J. (2006) Risk Factors for Postoperative Nausea and Vomiting. Anesthesia \& Analgesia, 102, 1884-1898. http://dx.doi.org/10.1213/01.ANE.0000219597.16143.4D

[12] Chimbira, W. and Sweeney, B.P. (2000) The Effect of Smoking on Postoperative Nausea and Vomiting. Anaesthesia, 55, 540-544. http://dx.doi.org/10.1046/j.1365-2044.2000.01474.x

[13] Apfel, C.C., et al. (1999) A Simplified Risk Score for Predicting Postoperative Nausea and Vomiting: Conclusions from Cross-Validations between Two Centers. Anesthesiology, 91, 693-700. http://dx.doi.org/10.1097/00000542-199909000-00022

[14] Sinclair, D.R., Chung, F. and Mezei, G. (1999) Can Postoperative Nausea and Vomiting Be Predicted? Anesthesiology, 91, 109-118. http://dx.doi.org/10.1097/00000542-199907000-00018

[15] Cepeda, M.S., et al. (2003) Side Effects of Opioids during Short-Term Administration: Effect of Age, Gender, and Race \& Ast. Therapeutics, 74, 102-112.

[16] Pusch, F., Berger, A., Wildling, E., Tiefenthaler, W. and Krafft, P. (2002) The Effects of Systolic Arterial Blood Pressure Variations on Postoperative Nausea and Vomiting. Anesthesia \& Analgesia, 94, 1652-1655. 